\title{
Memória e a diáspora japonesa no Canadá: Obasan, de Joy Kogawa
}

\section{Memory and Japanese diaspora in Canada: Obasan, by Joy Kogawa}

\author{
Maria do Rosário Silva Leite ${ }^{1}$ \\ Liane Schneider ${ }^{2}$
}

Submetido em 27 de março e aprovado em 25 de abril 2020.

Resumo: O presente artigo discute, no romance Obasan, de Joy Kogawa, o papel da memória, direta ou indiretamente acessada, como busca por inserção transcultural por parte de indivíduos no entrelugar canadense. Através da análise da composição de Naomi, protagonista nipo-canadense da narrativa em análise, bem como da discussão de conceitos de memória e transculturalidade, indicamos como as práticas culturais herdadas pelos primeiros migrantes que estabeleceram essa diáspora enriquecem o diálogo e o pertencimento de sujeitos marcados por mais de uma cultura no espaço contemporâneo canadense. Apontamos que o acesso a informações, através de recuperação da memória familiar, pode ser a ferramenta para que o conflito identitário possa ser tratado de forma mais positiva, tanto ficcional quanto comunitariamente.

Palavras-chave: Memória. Transculturalidade. Literatura Nipo-Canadense.

\begin{abstract}
This article discusses, in the novel Obasan, by Joy Kogawa, the role of memory, directly or indirectly accessed, as a search for transcultural insertion by individuals in Canadian in-between places. Through the analysis of Naomi's writing, the main Japanese-Canadian character of the narrative under analysis, as well as the discussion of concepts of memory and transculturality, we indicate how the cultural practices inherited by the first migrants who established this diaspora enrich the dialogue and the feeling of belonging of subjects marked by more than one culture in contemporary Canadian space. We point out that access to information, through the recovery of family memory, can be a tool so that identity conflicts can be treated in more positive way, both in fiction and as part of community life.
\end{abstract}

Keywords: Memory. Transculturality. Japanese Canadian literature. 
A memória é a identidade em ação.

Joël Candau

Cultural memory is thus the deeply-rooted quality that survives the impact of historical forgetting.

Benzi Zhang

\section{Introdução}

As políticas de imigração no que se refere aos povos asiáticos que se fixaram em território canadense implicaram uma série de medidas discriminatórias, com base na raça e etnia, promotoras de um ambiente sócio-político-cultural hostil e que se refletiu e reflete nos escritos de várias gerações, tanto de imigrantes quanto de seus descendentes nascidos em solo canadense. Discutimos nesse texto tais aspectos a fim de revisar a formação da literatura dos povos asiáticos produzidas no Canadá e em língua inglesa, da própria CanLit e da História literária canadense, já que indubitavelmente a presença asiática se tornou significativa na composição cultural do país.

Desse modo, nos propomos a discutir questões que envolvem o conceito de memória no romance Obasan (1983), de Joy Kogawa. Dentro do reconhecimento da diversidade cultural por parte daquele país, principalmente ocorrido nas décadas de 60 e 70, os povos asiáticos canadenses reivindicaram também um espaço literário para a representação de suas vozes, parte fundamental na construção histórica da nação canadense e de seu legado literário.

Desse modo, a literatura asiático-canadense não é simplesmente uma subseção multicultural de algo maior denominado literatura canadense. É mais relevante do que apenas um fragmento do quebra-cabeça literário. Ao invés disso, essa produção literária situa a literatura canadense dentro de uma rede global complexa e delicada constituída por rotas e passagens que são ao mesmo tempo históricas e culturais (CHO, 2008, p. 196)

$\mathrm{Na}$ verdade, reconhecendo o trajeto galgado pelos povos asiáticos, verificaremos o reflexo dos problemáticos enclaves étnicos e as negociações das múltiplas dimensões diaspóricas que acometem a protagonista do romance de Kogawa em análise, trazendo 
à luz o contexto nipônico canadense pela escrita de uma autora. Na realidade, ao abordarmos a escrita de autoria feminina no contexto das diásporas asiáticas. Como veremos a seguir, a narrativa de Kogawa torna-se representante de um compromisso com histórias de deslocamento e vivências transculturais relacionadas, dentro do legado da escrita migrante asiático-canadense de autoria feminina, elemento esse marcante na cena literária canadense contemporânea.

\section{O contexto de Obasan e a diáspora japonesa no Canadá transcultural}

A transculturalidade é negociada por Kogawa à luz das experiências migratórias em suas afinidades e disparidades, materializando-as em narrativas da memória, principalmente com respeito ao romance Obasan. Nesse, Naomi Nakane, uma professora primária de trinta e seis anos, narra a experiência do internamento (deslocamento da costa para o interior do país) sofrido pela mesma, ainda pequena, bem como por sua família e, mais amplamente, pelos japoneses no Canadá durante a Segunda Guerra Mundial. No decorrer do romance acompanhamos o desenvolvimento de Naomi da infância à fase adulta, os efeitos do pós-guerra, os conflitos entre gerações verificáveis nos diálogos com suas tias e os silêncios que rondam a história aos poucos desvelada por ela. Concomitantemente, essa narrativa avulta e abre espaço para novas leituras não apenas das vicissitudes do dia-a-dia nas comunidades diaspóricas, mas também com relação à posição da mulher no mundo globalizado. Esse olhar sobre as dimensões diaspóricas e suas experiências em relação à Naomi são definidas, em afirmação com o que aponta HALL (1990, p. 119-120):

pelo reconhecimento de uma heterogeneidade e diversidade necessárias, por via de uma concepção de identidade que subsiste com e através, não a despeito, da diferença; pelo hibridismo. Identidades diaspóricas são aquelas que estão constantemente em produção e reprodução de novas maneiras, por intermédio da transformação e da diferença. ${ }^{4}$

Nesse sentido, a protagonista plasma seu eu no decorrer da narrativa demonstrando os meandros das afiliações transitórias pelas quais é acometida ao longo das negociações culturais demandadas pela condição migrante e por seu gênero. Conforme apresentado, o romance lida com os aspectos relativos à mobilidade, seja essa interna, como decorrente 
do internamento no século XX dos nipo-canadenses, relatado em Obasan. Como estamos tratando de deslocamentos e trânsitos culturais vinculados a culturas tradicionais enraizadas fortemente em processos racializados, constatamos que a narrativa se insere, assim, dentro de um contexto diaspórico que se abre às experiências dos indivíduos e suas subjetividades. Conforme ressalta Coral Ann Howells (2009), a vantagem atribuída ao termo "diaspórico" é que esse sugere uma abordagem pluralística de reconhecimento étnico, cultural e racial da diversidade presente no Canadá. Segundo destaca Lily Cho (2007, p. 13) "voltar-se para a diáspora demonstra uma pretensão, no intuito de encontrar uma maneira para falar sobre as complexidades das conexões entre comunidades, das dores irreparáveis e pertencimentos desarticulados dos quais as coletividades emergem". 5 Desse modo, a protagonista deve lidar com os entraves étnicos estabelecidos pelas estruturas de poder, englobando as problemáticas que envolvem a perspectiva feminina em relação a sua representação e formação dentro de comunidades diaspóricas. Conforme proposto por CHO (2007, p. 28), "voltar-se para a diáspora é voltar-se para a influência das conexões e para a habilitação das possibilidades da diferença. Voltar-se para a diáspora é afastar-se da marcha aparentemente inexorável da história e ir em direção aos segredos das memórias incorporadas intimamente à convivência diária". ${ }^{6}$ Por conseguinte, as narrativas diaspóricas envolvendo as experiências de violência, traumas e chagas do passado, se tornam, então, desafiadoras e reconfiguradoras das histórias impostas pelo olhar dominante, assim como do armazenamento memorial de vidas anteriormente invisíveis.

Segundo destacam Bliss S. Little e Benjamin J. Broome (2010, p. 222),

(...) desde o final da década de sessenta, a diáspora tem sido usada para designar diferentes grupos de pessoas, incluindo minorias étnicas e raciais [...]. Além de se referir a grupos de pessoas, o termo diáspora tem sido utilizado, também, para aludir a dispersão de uma língua ou cultura ou qualquer objeto que é disseminado a partir de sua origem ou centro. ${ }^{7}$

Este trânsito cultural que engloba os diversos aspectos que envolvem as origens de um grupo não deve nos levar a teorizar as diásporas apenas a partir de vínculos, sejam estes impositivos ou não, que associam os indivíduos à coletividade. Stéphane Dufoix (2008, p. 2) defende que “[...] a menos que a pessoa se satisfaça com uma realidade 
estatística simples, atemporal e unificadora, a diáspora geralmente falhará nas tentativas de mostrar as engrenagens da coisa que ela deveria descrever melhor: a relação com o que chamo de 'origem de referência'" ${ }^{8}$ Portanto, a complexidade sempre deve fazer parte de qualquer olhar que se debruce sobre uma diáspora específica, a fim de não cometer erros por simplificação exagerada nos arranjos que marcam tais culturas, povos, grupos, formas de vida.

Consideramos fundamental destacar que a formação da literatura asiáticocanadense se deu pelo véu da racialização e etnicidade, vistas como pólos, a princípio, de exclusão, sujeitos a uma classificação imposta pelo olhar dominante, tendenciosamente homogeneizador. Em contrapartida, a racialização do termo Asian encarna os aspectos de uma história opressora e discriminatória que se abre posteriormente aos processos de negociação das partes integrativas da nação e da CanLit como amálgama de várias culturas, pois o termo asiático-canadense refere-se a um conjunto de povos reunidos por laços de contestação à civilidade branca. Em seu trabalho sobre a diáspora canadense, Lily Cho afirma que:

\begin{abstract}
A minoria marca uma relação definida pela racialização e vivenciada como diáspora. É assim que a literatura asiáticocanadense, por exemplo, é referida como uma literatura menor em relação à literatura canadense; não porque seja menos importante, valiosa ou esclarecedora, mas porque não pode ser dissociada das duradouras histórias de racialização que marcam as comunidades asiático canadenses como parte de uma minoria em relação à cultura dominante. Nesta experiência de racialização, comunidades asiático-canadenses estão marcadas tanto por um sentimento forte de pertença a este país, por ter ajudado a construí-lo, e por histórias de deslocamento que sustentam um sentimento de pertença que excede o Estado-nação (CHO, 2007, p. 98) $)^{9}$.
\end{abstract}

Dessa forma, os processos e intempéries oriundos das diásporas no Canadá direcionam e acompanham a história dos povos asiáticos contemplando o soerguimento dos afetos cultivados pelos deslocamentos e travessias de uma cultura para a outra, se fazendo presente através dos movimentos sociais e comunidades representativas da diversidade étnica existente no país. De fato, a literatura asiático-canadense emergiu acompanhada de variados ativismos comunitários, esses, por sua vez, baseados num corpo 
coletivo de identidades marcadas pela exclusão histórica. Nesse sentido, ao enfatizarmos os acontecimentos provenientes das migrações e assentamento desses povos, retomamos a memória histórica de um racismo velado, pois a narrativa em tela apresenta traços das imposições raciais, assim como os efeitos decorrentes dos discursos de diferenciação oriundos pós implementação das políticas multiculturais e sob o que denomina Charles Taylor (1994) de "política do reconhecimento". Contudo, o sujeito emergente desse reconhecimento não se constrói apenas através dos encontros interculturais, mas também no espaço do imaginário.

\section{Adentrando Obasan: memória na diáspora}

No âmbito ficcional, Obasan apresenta uma protagonista, Naomi, nascida no Canadá e representante da terceira geração (sansei) na linhagem nipônica, cuja família sofreu no passado as intempéries de um deslocamento forçado no território canadense, acompanhado do despojamento de bens e direitos, após o bombardeio a Pearl Harbor, fator esse que culminou com a exclusão e banimento do povo nipônico. Assim disposta, a narrativa resgata os acontecimentos que se desdobram entre os períodos da infância até a fase adulta da protagonista, recorrendo à memória cultural dos grupos em que essa se insere numa narração não linear. Segundo Benzi Zhang (2008, p. 87) essa memória histórico/cultural emerge "nem sempre como uma entidade de consistência ou imanência, mas como um domínio que multiplica conexões e absorve novos significados. [...] como resultado, deve ser considerada um devir que luta para recuperar novas relações entre o passado e o presente". ${ }^{10}$ Nesse sentido, podemos verificar, nos relatos de Naomi, a chamada memória episódica, segundo denomina Sharon Selby (2018, p. 8), que consiste em memórias de um indivíduo que ocorreram através do tempo, um tempo esse específico de sua jornada e que são consideradas "a sede da subjetividade". ${ }^{11}$ É por meio, também dessa memória que Naomi faz seu relato das injúrias raciais por ela sofridas e que são reflexo de todo um coletivo nipônico canadense.

Em Obasan, as imposições raciais, assim como as leis de cunho racista, se tornam visíveis em muitas das cartas de tia Emily mencionadas ao longo da narrativa (o que se verifica também em documentos oficiais correspondentes ao período), onde a seguinte mensagem fica clara: "chutem todos os japas para fora" (KOGAWA, 1983, p. 102). ${ }^{12}$ 
Apenas no último minuto, antes de tia Emily se encaminhar para o embarque, ela me perguntou se eu realmente queria "saber de tudo". "Eu vou enviar-lhe um pouco da minha correspondência e outras coisas. Você gostaria?", perguntou ela.

"Claro", eu menti.

Agora estou aqui nesta manhã de outono, cinco meses depois, com seu pacote pesado no meu colo. Muitos dos textos de conferências que ela me mostrou em maio passado estão aqui, bem como o seu diário e um envelope pardo bojudo cheio de letras. [...] Obasan se agacha para pegar as cartas e as entrega abertas para mim, uma de cada vez.

Há uma cópia de uma carta de Mackenzie King; uma para o Sr. Glen, o ministro de Minas e Recursos; uma do Comandante do Quartel-General Supremo das Forças Aliadas; [...] Todos os detalhes da morte que são deixados nos colos dos vivos (KOGAWA, 1983, p. 43). ${ }^{13}$

A leitura de Naomi, ainda que relutante, aos poucos a encaminha para o diário de sua tia, "este diário da tia Emily é o maior que eu já vi”" (KOGAWA, 1983, p. 79). ${ }^{14}$ As páginas não são numeradas e se trata de um diário de cartas destinadas à mãe de Naomi, sendo que ali são relatados os acontecimentos do internamento e seu reflexo nas famílias Nakane e Kato entre os anos de 1941 e 1942, como lemos: "o livro se torna pesado com as vozes do passado - uma conexão da mamãe com a vovó Kato que eu não sabia que existia" (KOGAWA, 1983, p. 46). ${ }^{15}$ Ao se apropriar das narrativas pretéritas, a protagonista de Kogawa está a princípio receosa quanto à leitura, “eu me sinto como uma ladra quando leio, arrombando uma casa particular apenas para descobrir que é a casa da minha infância cheia de cantos e cômodos que eu nunca vi” (KOGAWA, 1983, p. 79). ${ }^{16}$ A protagonista descobre novas perspectivas sobre o seu passado e lê as cartas através das lentes da experiência migrante. Segundo a visão de Naomi, as cartas e o diário se interligam representando uma "semente da rememoração a este conjunto de testemunhos exteriores [...], uma consistente massa de lembranças" (HALBWACHS, 2006, p. 32). Após a leitura do diário de sua tia Emily, Naomi sente uma instabilidade e desorientação, na realidade, efeito catalisador dos processos memoriais iniciados por sua tia Ayako durante a visita ao sótão, depois expandido por Emily e sua memória epistolar. Vejamos o fragmento:

Às vezes eu não estou certa se é um sótão desordenado no qual eu me sento, uma sala de espera, um túnel, um trem. Não há começo 
nem fim para a floresta, ou para a tempestade de poeira, não há limite a partir do qual se possa saber onde a clareira começa. Aqui, nesta densidade familiar, sob este manto, dentro desta carapaça, há o anseio dentro da escuridão" (KOGAWA, 1983, p. 111). ${ }^{17}$

Desse modo, o seu interior desperta após anos de um silêncio protetivo, que se metamorfoseia e induz à mobilidade num interminável espaço, sem demarcações que auxiliem na compreensão dos fragmentos que a mesma tenta juntar a fim de reconstruir sua história através das vozes de sua mãe, de sua tia Emily e avó Kato (que acompanha a mãe de Naomi até o Japão). As cartas enviadas por sua avó Kato, em 1949, complementam a compreensão que Naomi tem sobre os fatos até então resguardados de seu entendimento, descrevendo detalhes da explosão atômica em Nagasaki e as consequências sobre a mãe de Naomi. Por meio das cartas da avó, a protagonista ouve ecoar a voz de sua mãe que transforma a estase de suas ambivalências interiores em movimentos livres.

Se a nossa impressão pode se basear não apenas na nossa lembrança, mas também na de outros, nossa confiança na exatidão de nossa recordação será maior, como se uma mesma experiência fosse recomeçada não apenas pela mesma pessoa, mas por muitas. [...] os fatos passados assumem importância maior e acreditamos revivê-los com maior intensidade, porque não estamos mais sós em representá-los para nós. Não os vemos agora como os víamos outrora, quando ao mesmo tempo olhávamos com os nossos olhos e com os olhos de um outro (HALBWACHS, 2006, p. 29-30).

Portanto, nossa protagonista, inserida numa sociedade diversa e multifacetada como a canadense, adquire uma voz cada vez mais revisionista de sua história. Naomi busca problematizar sua localização nas duas culturas que a marcam. Segundo BHABHA (1998, p. 20) "esses entre lugares fornecem o terreno para elaboração de estratégias de subjetivação - singular ou coletiva - que dão início a novos signos de identidade". Sendo assim, a identidade de Naomi é construída primeiramente entre dois polos de resistência - o silêncio e o som, ambos representados respectivamente por suas tias Ayako e Emily. Nesse sentido, as vozes femininas em Obasan induzem a rememoração e agenciam as negociações da protagonista como forma de revisão do próprio eu ficcional.

Naomi partilha das experiências constituintes da vivência Issei (primeira geração) com o tio Isamu, a tia Ayako e sua avó Kato, que são ao mesmo tempo contrabalanceadas 
pela convivência com o irmão Stephen e sua tia Emily, ambos mais vinculados aos processos de assimilação à cultura de chegada. Principalmente por meio do contato com sua tia Ayako, a personagem tem a oportunidade de aprender e manter alguns costumes da cultura japonesa, a exemplo do banho como ritual ${ }^{18}$ familiar, partilhado por Naomi com sua avó Kato e, após o internamento, com sua tia Aya, no balneário da cidade. De acordo com Anne-Marie Lee-Loy (2010, p. 324):

(...) banhos públicos e tomar banho tem uma especial ressonância cultural para os japoneses, não apenas como uma questão de higiene, mas porque balneários são entendidos como locais em que os valores culturais e de socialização podem ser praticados e um "pensamento grupal" culturalmente mais específico se expressa. ${ }^{19}$

O banho se torna uma imersão cultural nos costumes ancestrais e uma forma de afeição dividida. Na casa em Vancouver, Naomi partilha com sua avó Kato desse ritual. Entretanto, a protagonista só participa dele no intuito de alegrar a avó. O sentimento expresso por Naomi divide-se entre aceitar aquele costume e vivenciá-lo como forma de partilha, representando unidade familiar e étnica, como lemos, "nós somos uma única carne, uma família, lavando uns aos outros ou estando submersos em água quente" (KOGAWA, 1983, p. 160) ${ }^{20}$; ou vivenciando o banho como um sacrifício, "doce sacrifício", em favor da satisfação alheia, denominado sasshi.

O conceito do termo enryo-sasshi remete aos estudos relacionados a teoria da comunicação de Satoshi Ishii (1984) ${ }^{21}$, indicando que os termos enryo and sasshi correspondem respectivamente ao autodomínio e a percepção/intuição, que segundo o autor, tratam de estilos de comunicação frequentemente presentes na cultura nipônica. Anterior a Ishii, temos Takie Lebra cujo livro Japanese patterns of behavior, de 1976, trouxe à lume, através da antropologia, um maior entendimento à respeito da cultura nipônica, destacando assim o conceito enryo-sasshi. Ambos os termos, de acordo com Takie Lebra (1976), são ao mesmo tempo a supressão de uma individualidade perante a pressão exercida pela comunidade como forma de criar uma atmosfera de solidariedade e conforto, esses expressos pela contenção de ideias e sentimentos, uma forma de garantir a harmonia social. Em relação ao enryo o teórico esclarece: 
Enryo é um produto da supressão da individualidade sob a pressão da solidariedade e conformidade do grupo, considerações empáticas pela conveniência ou conforto de outros, preocupação em evitar o constrangimento do próprio ego e o desejo de manter a liberdade desse, evitando o envolvimento social sem magoar (LEBRA, p. $252,1976){ }^{22}$

No romance em análise esses conceitos, tidos como basilares na cultura japonesa, se entrelaçam e aparecem vinculados ao comportamento de Naomi, influenciando-a na relação com as culturas que a marcam. Nesse sentido, essa linha divisória estabelecida pela protagonista sinaliza mais uma vez o entre lugar que essa ocupa no que se refere a seus elos, seus vínculos e pertencimentos. Tal negociação e participação no ritual do banho são levados a cabo por Naomi por respeito à avó, já que seu irmão Stephen se esquiva do banho familiar coletivo. Enquanto Stephen busca, dessa forma, demarcar sua total posição de recusa à vivência nipônica, Naomi utilizava o momento para aproximarse dos mais velhos.

$\mathrm{Eu}$ agachei lentamente na outra extremidade da banheira. Isso é uma doce tortura, com a vovó feliz, aprovando e apreciando o calor que eu mal posso suportar. Eu sou mais corajosa e mais louvável do que o Stephen. Ele não vai tomar banho com a vovó. Mas eu vou sofrer as indignações sem fim da carne para o prazer dela. [...] O meu corpo está estendido ao lado dela e ela faz ondas para cobrir meus ombros. Uma vez que o corpo está totalmente imerso, há uma paz entorpecida [...]. Em algum momento, a vovó abriu os olhos e enrolou sua toalhinha num mãe firme e úmida. Eu permaneço ao lado dela e sobre a vermelhidão do meu corpo, ela esfrega vigorosamente, como uma borracha sobre a página suja. [...] Ela esfrega cada um dos meus dedos, minhas mãos, braços, peito, barriga e abdômen, pescoço, costas, nádegas, coxas, pernas [...] ela me ensaboa completamente e eu me lavo antes que ela ensaboe a si mesma. [...] Eu estou vestida com um nemaki, uma peça de roupa de dormir de algodão roxo e branco, que a vovó costurou à mão. Tem mangas em forma de envelope retangulares com aberturas nos axilas e dois laços reunidos em uma curva na parte de trás. Estou extremamente segura no meu nemaki, sob o futon pesado de cores claras na minha casa (KOGAWA, 1983, p. 48). ${ }^{23}$

Num segundo momento, Naomi e Ayako, sua Obasan, partilham do banho comunal, após o internamento, no final da noite na cidade de Slocan. Conforme destaca LEE-LOY (2010, p. 324) ao comentar essa passagem do romance, "de fato, a representação mais célebre do banho é o silencioso balneário em Slocan, no qual Naomi e Obasan são rejeitadas 
pelas outras mulheres da comunidade. Significativamente, neste episódio, o silêncio realmente converge com a prática cultural para fragmentá-la, em vez de fortalecer uma identidade étnica" ${ }^{24}$ Como descrito na narrativa, o local já se encontra quase desocupado, permanecendo ali apenas duas mulheres e duas crianças. Como habitualmente, Naomi e Aya seguem a rotina na casa de banho, mas são observadas pelas outras. Naomi reconhece uma das crianças, e a cumprimenta, mas a mesma é repreendida pela mãe para evitar conversas, "quando eu olho para trás, ela me reconhece com um sorriso torto e hostil, no entanto continua acenando para as meninas. Por alguma razão ela me desaprova" (KOGAWA, 1983, p. 162). ${ }^{25}$ Naomi observa atentamente as duas mulheres, enquanto sua tia e Nomura Obasan continuam com as cabeças reclinadas, evitando o contato visual direto, aspecto esse, parte da cultura nipônica e que não é seguido pela protagonista, que mantém seu olhar enfocado,

as duas mulheres estão cochichando com Reiko e Yuri evitando qualquer contato conosco, nenhuma saudação formal ou informal. Obasan e Nomura obasan mantêm suas cabeças igualmente abaixadas. Yuri, que estava nadando ao redor e me chamando, para e me olha de forma estranha. "O que, eu me pergunto, pode ser?" (KOGAWA, 1983, p. 163). ${ }^{26}$

O ritual de socialização torna-se local de rejeição social para Naomi e sua tia, devido à notícia na comunidade de que seu pai e irmão estão com tuberculose (TB). Além da doença ser contagiosa, a mesma é considerada um constrangimento social, segundo o preceito hazukashii. ${ }^{27}$ A princípio Naomi não compreende o motivo do afastamento, apenas percebe o olhar cabisbaixo e gestos evasivos dos que as cercam, "eu permaneço no canto sozinha, vendo as duas mulheres cochichando com os olhos cabisbaixos" (KOGAWA, 1983, p. 162 ${ }^{28}$. Segundo a tradição japonesa dos banhos, a saúde é objetivo primordial e de valor elevado. Nesse sentido, a doença de seus familiares reflete negativamente na família e em sua posição dentro da comunidade. A família é vista, então, como perigosa, já que pode comprometer a saúde de todos na comunidade. A exclusão apresenta, dessa forma, uma desarticulação de laços étnicos, pois seria por meio do compartilhamento de costumes, isso aliado ao uso da língua que esses laços seriam ratificados. Nesse sentido, da exclusão, têm-se um distanciamento que se estendeu primeiramente do espaço macro 
(com o internamento) no qual todo um coletivo foi repelido, para o micro (com as comunidades) criando novas distâncias sociais.

No romance de Kogawa, o tom introspectivo da narrativa é interrompido pelo ativismo político da tia Emily, que rompe com o silêncio de Ayako (Obasan) a fim de fazer com que Naomi (re)conheça seu passado. Tia Emily, segundo Naomi, "é uma guerreira da palavra" (KOGAWA, 1983, p. 32) ${ }^{29}$, propensa a coordenar protestos e a fazer uso da fala em prol da causa nipônica ou de qualquer outra causa que lhe pareça justa. Persistente em manter sua identidade política como cidadã canadense, é através da fala e escrita de Emily que a dimensão histórico-cultural das injustiças contra os nipo-canadenses são apresentadas e agregadas à narrativa de Naomi. Vinculada ao "som" (e não ao silêncio), tia Emily, diferentemente de Ayako (obasan), está mais propensa a seguir os hábitos da cultura ocidental, ou seja, está afinada com a cultura canadense, de modo que Emily não consegue mais acesso aos registros culturais provenientes do silêncio, como lemos: "pelo resto da viagem de carro até em casa eu me mantive calada, enquanto tia Emily nos intimidava. Eu pude perceber que estávamos nos encaminhando para uma noite de discussões, independentemente de qualquer um querer ou não" (KOGAWA, 1983, p. 35). ${ }^{30}$

Identificada primeiramente pela forma como Naomi a chama, sempre tia Emily (aunt ${ }^{31}$ Emily), a palavra Obasan, que significa "tia" em japonês, nunca é aplicado em relação à Emily. Além disso, a ocidentalização de seu nome (Emily) e comportamento destoante dessa em comparação aos parentes, pelo seu ativismo barulhento, endossam uma vivência com características já mais culturalmente mescladas, não apenas afinada com os aspectos culturais nipônicos. Isso é ressaltado na comparação feita por Stephen, irmão de Naomi, sobre Emily e seus tios Isamu e Ayako, ““'ela não é como eles”, Stephen diz, sacudindo o polegar para o tio e a tia" (KOGAWA, 1983, p. 215). ${ }^{32}$ Contudo, vale considerar que socialmente por dominar as ferramentas do discurso no estilo mais canadense mainstream é que Emily consegue vociferar e reclamar pelos direitos, inclusive dos parentes. Emily não segue alguns parâmetros da cultura nipônica e que são repassados por Ayako à Naomi, a exemplo do enryo, quinta-essência do comportamento japonês, que tem por significado ser modesto, reservado, que, "funcionando como um filtro psicológico, requer o controle dos pensamentos e ações da pessoa como forma de 
evitar falar ou fazer coisas irrefletidamente" (MIIKE, 2010, p. 250) 33 ; também se afasta do que é denominado yasashi (gentil), uma das qualidades relembradas por Naomi e que aparece vinculada à sua mãe, "a voz da mamãe é yasashi, macia, suave no escurecer da luz do dia. Ela é inteiramente yasashi” (KOGAWA, 1983, p. 51). ${ }^{34} \mathrm{O}$ termo é associado a conduta sasshi a qual, segundo MIIKE (2010), demanda que o indivíduo seja perceptivo às necessidades alheias como demonstração de uma sensibilidade social. No romance Ayako também ensina Naomi a não ser wagamama (egoísta) e a possuir uma conduta sasshi, "Obasan me ensina a não ser wagamama destacando constantemente as necessidades dos outros" (KOGAWA, 1983, p. 128) ${ }^{35}$ A convivência com tia Emily é interrompida em maio de 1942, data na qual Stephen, Naomi e Aya (Obasan) partem de trem para a cidade de Slocan, reencontrando com Emily apenas doze anos mais tarde. No entanto, dois anos antes, em 1952, Stephen embarca para Toronto a fim de morar na casa de Emily após vencer um importante festival de música em Granton. Desse modo, trafegando entre as polaridades indicadas e fomentadoras de outros espaços, Naomi se encontra, assim, imersa em suas memórias como forma de ler os silêncios e os sons através das lentes racializadas e culturalmente flexionadas da experiência nipo-canadense.

Dessa forma, Naomi, através da narrativa em primeira pessoa, tenta compreender o seu passado como forma de lidar com seu presente, adentrando novos domínios em seu reservatório de memórias, de modo a transcender estereótipos, dialogando com a cultura ancestral e de chegada, em um ambiente transcultural, diferentemente de seu irmão Stephen, que se afasta de qualquer relação direta com a cultura nipônica, "Stephen se tornou completamente estranho para com a língua japonesa" (KOGAWA, 1983, p. 231) ${ }^{36}$ Stephen se afasta não apenas da língua, mas também evita vários alimentos tipicamente nipônicos, como o onigiri (bolinho de arroz), negando a compreensão de pequenos poemas japoneses (haiku) ou qualquer outra marca étnica ou mesmo sinais diacríticos que possam endossar o seu fenótipo, buscando extirpar esse pertencimento cultural.

Segundo Philippe Poutignat e Jocelyne Streiff-Fenart (2011, p. 168) “à medida que, numa sociedade pluralista, os indivíduos conhecem a existência e o conteúdo dos estereótipos que os outsiders têm sobre eles, esses orientam-se no mundo de estereotipia tentando afastar os realces que lhe são perigosos e promover aqueles que são vantajosos". 
É exatamente o que ocorre com Stephen, que se sobressai em sua carreira musical como forma de enaltecer os atributos que lhe favoreçam perante a cultura dominante. Por outro lado, Naomi permanece numa posição diferente da adotada pelo irmão. Enquanto esse adere ao apagamento das marcas étnicas no intuito de se tornar "mais canadense", ela se interessa pelas influências múltiplas, não pretendendo e nem conseguindo pertencer a apenas um lado. Nesse contexto, podemos considerar a posição de Naomi como a do entre lugar, diferentemente da forma como seu irmão e mesmo sua tia Emily agem nas relações com a sociedade canadense hegemônica, reprimindo suas origens como forma de se inserir na cultura de chegada. A própria tia Emily afirma a posição ocupada por Naomi nesse entre lugar, destacando que a mesma fora alimentada com leite e pela história do Momotaro (o menino pêssego), “"“que bebê mais sério - alimentado com leite e Momotaro". "Leite e Momotaro?" Eu perguntei. "Choque cultural?"” (KOGAWA, 1983, p. 57). ${ }^{37}$ A questão do leite estaria atrelada a uma vivência geralmente não pastoril dos japoneses, o que consequentemente os inclui dentre os povos intolerantes a lactose ${ }^{38}$. No caso de Naomi o consumo do leite, sem maiores problemas, agregado ao embalar da história tradicional japonesa do Momotaro, marcaria sua melhor negociação entre as culturas que lhe são intrínsecas.

Em outra passagem do romance Obasan, a posição maleável que Naomi assume é contraposta ao "grupo assimilado", ou seja, a protagonista rememora sua experiência na escola em Granton, local no qual Stephen se destaca como o melhor aluno, recebendo prêmios por seu talento musical. Na verdade, o comportamento de Stephen e seus hábitos já condicionados pela assimilação da cultura dominante o tornam um sujeito mais fácil de ser incluído pela cultura de chegada, como observa Naomi, “ele fica sempre desconfortável quando alguma coisa é "muito japonesa"”, (KOGAWA, 1983, p. 217). ${ }^{39} \mathrm{Na}$ escola, Naomi recebe tratamento diferenciado, mas não num sentido positivo, como observamos no fragmento seguinte, onde a comparação demonstra que Naomi é marcadamente gendrada, racializada e etnicizada por não corresponder à assimilação, como faz seu irmão:

[...] nas classes sete e oito das aulas da Miss Langston, eu tenho vivido um tempo difícil para à reputação dele. Ele era seu aluno favorito. Às vezes me pergunto se por ela estar tão desapontada comigo, me avalia tão severamente. Seus X vermelhos em meus 
trabalhos são como arranhões e feridas. "Não é como seu irmão, é?

"Ela me disse certa vez, me retornando o insignificante trabalho" (KOGAWA, 1983, p. 202). ${ }^{40}$

Observamos, assim, que a diferença no tratamento implica numa dissociação entre canadenses e os "outros canadenses". O que se observa é que "as identidades são fabricadas por meio de sistemas simbólicos da marcação da diferença. Essa marcação da diferença ocorre tanto por meio de sistemas simbólicos de representação quanto por meio de formas de exclusão social" (WOODWARD, 2012, p. 40). Diante disso, Naomi, está alocada num espaço de redefinições, fator esse de estranhamento e tratamento diferenciado por parte do grupo hegemônico representado por sua professora. Se a escola é o local oficial de inserção social, certamente Naomi percebe que ali não encontra lugar confortável, sendo marcada por contradições.

Nesse contexto, o espaço ocupado por Naomi é marcado por um conceito de transculturalidade que "esboça uma imagem diferente da relação entre culturas. Não uma de isolamento e de conflito, mas uma de enredamento, miscigenação e generalidade. Promove, não a separação, mas a troca e interação" (WELSCH, 1999, p. 205). ${ }^{41}$ Isso não quer dizer que os conflitos ou as divisões deixem de existir, pelo contrário, a leitura de Welsch propõe que os encontros culturais não se pautam apenas pelos embates, mas que, a partir da eclosão de sobreposições culturais e distinções se possa promover um maior espaço de negociação.

\footnotetext{
“Trans", em relação às culturas, refere-se também a reconhecer os valores e propósitos comuns que "transcendem" as margens de culturas particulares. Transculturalismo envolve olhar para si mesmo no disfarce cultural, através dos olhos dos outros. Esse processo, ao passar em circunstâncias de respeito mútuo, não deixa de implicar num grau de libertação das imutabilidades culturais, a partir da ideia de mutuamente realizar e materializar múltiplas identificações. [...] o vínculo em sociedades heterogêneas deve envolver negociação em curso e revisões de noções de direitos coletivos e individuais, assim como responsabilidades entre os grupos dominantes e dissidentes (ESSED, 2008, p. 242). ${ }^{42}$
}

Dessa forma, em face às narrativas históricas de deslocamentos, trânsitos e travessias desencadeadoras das formações diaspóricas e consequentemente dos imbricamentos e negociações culturais, as quais inseriram marcas indeléveis na constituição da nação 
e letras canadense, verificamos a emergência das vozes racializadas que os processos migratórios conduziram gradativamente das margens para o centro, despertando-as do repouso dogmático para assim inseri-las através da "quebra do silêncio" e da resiliência dos povos marginalizados pela supremacia branca no contexto de expansão multiculturalista. Esses povos condensam, em seu espaço social, narrativas multiaxiais profundamente enraizadas nos aspectos raciais.

Visualmente identificados como "estrangeiros" e submetidos, portanto, à racialização, os membros das "minorias visíveis" nestas estórias problematizam definições dominantes da nação multicultural canadense, que ainda no século XXI, se apoia fortemente em seu passado colonial. (DOMÍNGUEZ; LUCAS; LÓPEZ, 2011, p. 43). ${ }^{43}$

Essa perspectiva adotada no romance apresenta, pela vivência da protagonista, a perspectiva de mulheres marcadas por sua dupla condição marginal representada através de um passado abusivo e um presente de exclusão na sociedade de chegada. A protagonista atravessa as intempéries da condição migrante marcada por seu gênero, e, obviamente, também pelo lugar social que seu grupo étnico tende a assumir.

No romance de Kogawa, tanto Naomi como os nipo-canadenses em geral naquele período histórico foram condenados a uma marcha país adentro e ao isolamento como uma tentativa de apagamento da presença japonesa no país. A exclusão sentida a princípio por seu irmão é transmitida para Naomi como um primeiro questionamento identitário, "a garota com longos cachos que se senta na frente do Stephen disse-lhe. "Todas as crianças japas da escola serão mandadas embora, eles são maus e você é um japa”. E assim Stephen me diz, que eu sou. "Nós somos papai? "Não," papai responde. "Nós somos canadenses". É um enigma, Stephen me diz. Nós somos ao mesmo tempo o inimigo e o não inimigo" (KOGAWA, 1983, p. 70). ${ }^{44}$ Posteriormente, após a perda dos bens e o recrutamento de seu pai e tio para os campos de trabalho, Naomi segue com parte da família para a cidade de Slocan. Na cidade, “todos os professores e crianças em nossa escola são japoneses. As crianças brancas em Slocan vão para uma escola diferente" (KOGAWA, 1983, p. 139). ${ }^{45}$ A experiência em Slocan configura em Naomi as diferentes posições atribuídas ao indivíduo tanto pela cultura de chegada quanto pela vivência dentro 
da comunidade nipo-canadense, através da experiência social de integração e rejeição. Esses sentimentos ambíguos são marcados pela fragmentação de Naomi, representados pelos estalidos das rodas do trem ao deixar a cidade, "pelas margens da cidade, seguindo, pelas árvores e para cima, ao longo das estreitas cordilheiras, por um túnel e estamos no nosso caminho novamente. Tchak, tchak, estalando, adeus, Slocan” (KOGAWA, 1983, p. 181$)^{46}$, passando por um túnel, que no romance indica "o caminho de uma iniciação" (CHEVALIER; GHEERBRANT, 2007, p. 915) com travessias inquietas e dolorosas “que podem desembocar em outra vida" (CHEVALIER; GHEERBRANT, 2007, p. 916), deslocando-se, mais uma vez, juntamente com a família, agora para Lethbridge, Alberta. Esse sentimento oscilante entre rejeição e aceitação está presente no romance em tela e acompanha a protagonista em suas múltiplas identificações, descritas como intermitentes fragmentações e suplementações.

Desse modo, de posse de um legado histórico e literário erigido com base nos fluxos migratórios, o tecido cultural da narrativa asiático-canadense em foco emerge como espaço de resistência e tem nas relações entre memória e identidade a formação de renovadas formas de representação, nas quais as personagens centrais trafegam numa via de mão dupla para com a tradição. Segundo destaca Daniel Coleman (2006) a literatura das minorias busca desconstruir a "normatividade branca" regente das relações sociais e mantenedora de resoluções racistas, que tende a se perpetuar, mesmo que sob o discurso multiculturalista responsável por empregar a configuração de um Canadá tolerante. Nesse sentido, "é obvio que pela própria organização de suas comunidades pela raça e em termos étnicos, o Estado cria constantemente "canadenses" e "outros" (BANNERJI, 2000, p. 72). ${ }^{47}$ A identificação racial, segundo destacam Randy Enomoto e Genevieve Fuji Johnson (2007, p. 13) acaba, pois:

Enquadrada pelo discurso do multiculturalismo, sob o qual a cor da pele é empregada para identificar indivíduos quer como canadense ou como 'Outro'. Nesta forma de racialização, os indivíduos recebem uma identidade racial pelos pais, identificados pelos membros da sua comunidade étnica ou racial, ou por membros da sociedade em geral. Eles, então, crescem para aceitar ou rejeitar esta identidade. Tais construções identitárias emergem como uma resposta ao racismo e à racialização para com aqueles identificados e submetidos como 'outros' canadenses. ${ }^{48}$ 
Sendo, pois, marcados pela visibilidade racial frequentemente vinculada ao preconceito e à exclusão social, a autora manifesta, através da criação ficcional, uma releitura resultante dos entrelaçamentos históricos geradores de memórias múltiplas associadas aos entrecruzamentos culturais das diásporas contemporâneas. De fato, as narrativas contemporâneas em "produções recentes intermediadas por escritores étnicos, multiculturais ou minoritários no Canadá tem se tornado mais diversificados e experimentais na forma, tema, foco e técnica" (TY; VERDUYN, 2008, p. 3). ${ }^{49}$ Dentro de um sistema de tensões no qual a "brancura foi naturalizada como norma para a identidade cultural do Canadense anglófono" (COLEMAN, 2006, p. 5) ${ }^{50}$, as práticas narrativas desses povos se pautam nas experiências diaspóricas e nas existências transculturais como forma de transformar a visão eurocêntrica que os marca negativamente. Nesse sentido, a narrativa em análise discute os processos identitários e de pertença, ultrapassando padrões binários, descentrando uma vivência pendular para torná-la aberta e indeterminada, pois Naomi está em negociação com seus possíveis eus, já que as “identidades são, pois, identificações em curso" (SANTOS, 1999, p. 135).

\section{Buscando conclusões}

Considerado um ponto de ruptura determinante e facilitador das possíveis articulações de histórias de violência e repressão, a "quebra do silêncio", conjuntamente à interrupção de uma história alheia às minorias racializadas, amplia os espaços da produção literária a partir das memórias de sobreviventes e/ou descendentes de momentos históricos de discriminação de povos diaspóricos. De acordo com Vijay Agnew (2008, p. 184-185) "a memória é uma chave para a história pessoal, social e cultural e é dependente do tempo e contexto. Aquilo que nós lembramos é articulado pelas grandes narrativas políticas e sociais de nosso tempo, nos permitindo reconstruir a história através da identificação, e preenchendo as lacunas em suas muitas narrativas" ${ }^{\prime 51}$. Como um dos elementos basilares nas reconstruções de uma consciência diaspórica, as memórias apresentadas na narrativa em análise implicam num contínuo processo de redescobrimento, responsável por reconectar passado e presente. Conforme assertiva de Edward Said (2011, p. 34) o passado carrega os espectros regentes de uma história diaspórica que se empenha em fincar raízes nos processos de racialização, de modo que, 
a invocação do passado constitui uma das estratégias mais comuns nas interpretações do presente. O que inspira tais apelos não é apenas a divergência quanto ao que ocorreu no passado e o que teria sido esse passado, mas também a incerteza se o passado é de fato passado, morto e enterrado, ou se persiste, mesmo que talvez sob outras formas.

Desse modo, em Obasan, o passado rege a configuração do self da protagonista, pois Naomi é marcada por uma vivência diaspórica que a convida a "viver nas fronteiras do presente" (BHABHA, 1998, p. 15) agregando novos valores e condutas ao seu modo de vida. Aqui a diáspora é compreendida "como uma condição de subjetividade" (CHO, 2007, p. 14) $)^{52}$ ou de pertencimento que não é atribuída pelo nascimento, mas sim, é decorrente dos efeitos de forças políticas, sociais e culturais influentes nas dimensões afetivas da vida "presente" de ambas. Dessa forma, verificamos que a narrativa convoca o passado como forma de desvendar a conduta impositiva exercida sobre a protagonista através da comunidade e sistema político, encetando a reflexão ou mesmo ações diretas que vão de encontro às práticas ditadas pelo patriarcado e pelo racismo. Conforme destaca Ahn Hua (2008, p. 198) "Estes atos memoriais de transferência envolvem a negociação dinâmica entre o passado e o presente, entre o individual e o coletivo, o público e o privado, entre recordar e esquecer, poder e impotência, história e mito, trauma e nostalgia, consciência e inconsciência, medos e desejos". ${ }^{53}$

Todos esses aspectos surgem como coparticipantes da reconstrução das histórias silenciadas por grupos dominantes. Conforme acompanhamos nos pontos anteriores, o contexto histórico sob o qual se alicerçou a presença asiática no Canadá procede de vários embates oriundos da questão racial seguida por outros fatores desencadeadores de uma visão negativa sobre esses povos. Naomi, por exemplo, não pôde entrar na casa de uma menina branca, em cuja fazenda seus avós trabalham. "Uma vez, quando à Penny me trouxe até à porta, sua mãe falou através de sua pequena e precisa boca, "Penny, eu te disse, eu te disse", e fechou a porta me deixando sozinha do lado de fora" (KOGAWA, 1983, p. 222). ${ }^{54}$ Aqui a pequena Naomi percebe que não é bem-vinda na casa da colega, nem na comunidade canadense em que busca um lugar para si. A exclusão sofrida por Naomi indica reações e rejeições vivenciadas por todo um coletivo nipônico revisitado através da protagonista de Kogawa, que desvela traços de um colonialismo vetusto, que continua à espreita. 
Desse modo, entendemos que a compreensão do indivíduo se estende para além dos aspectos unívocos para se entrelaçarem a patamares globais, no qual a "memória cultural ou coletiva constitui a memória coletiva de muitos, englobando gerações" (HUA, 2008, p. 198 $)^{55}$. Sendo esse entrelaçar realizado pela memória, no caso específica de nossa protagonista, uma memória que adquire caráter fluido, permitindo, assim, uma revisão do contexto das diásporas via processos que se alicerçam em diferenças e diversidade. A literatura asiático-canadense revela, assim a problematização identitária de sua protagonista, mantida num devir sempre postergado.

Nesse sentido, as narrativas produzidas por povos diaspóricos canadenses como o aqui trabalhado, voltando-se aos entrecruzamentos culturais, às noções de pertencimento, percepções étnicas e híbridas de uma história cultural transcultural revista pelo olhar migrante, redefinem os dos diversos lugares (étnicos, sociais, culturais) que compõem a sociedade canadense contemporânea. Assim, as narrativas demandam uma versão mais inclusiva e menos monolítica do que seria "ser canadense", já que a memória cultural do trauma, em nosso estudo, vivenciado pelos povos asiáticos, ainda ronda gerações de escritores daquele país.

\section{Referências}

AGNEW, Vijay (ed.). Diaspora, memory and identity: a search for home. Toronto: Canada: University of Toronto Press, 2008.

BHABHA, Homi. O local da cultura. Tradução Myriam Ávila; Eliana Lourenço de Lima Reis; Gláucia Renate Gonçalves. Belo Horizonte: UFMG, 1998.

CHEVALIER, Jean; GHEERBRANT, Alain. Dicionário de símbolos. 21 ed. Tradução Vera da Costa e Silva; Raul de Sá Barbosa; Angela Melim; Lúcia Melim. Rio de Janeiro: José Olympio, 2007.

CHO, Lily. Asian Canadian Futures: Diasporic Passages and the Routes of Indenture. In: FEE, Margery (ed.). Canadian Literature. Number 199, (Winter), Vancouver, Canada: University of British Columbia, 2008.

COLEMAN, Daniel. Writing dislocation. Transculturalism, gender, immigrant families: a conversation with Ven Begamudré, Canadian Literature 149, 1996.

DOMÍNGUEZ, Pillar Cuder; LUCAS, Belén Martín. LÓPEZ, Sonia Villegas. Transnational Poetics: Asian Canadian women's fiction of the 1990s. Ontario, Canada: TSAR Publications, 2011. 
DUFOIX, Stéphane. Diasporas. Translated by William Rodarmor. Berkeley: The University of California Press, 2008.

ESSED, Philomena. Multi-identifications and transformations: reaching beyond racial and ethnic reductionisms. In: JOHNSTON, Genevieve Fuji; ENOMOTO, Randy (ed.). Race, Racialization, and Antiracism in Canada and beyond. Toronto: University of Toronto Press, 2007.

HALBWACHS, Maurice. A memória coletiva. Tradução Beatriz Sidou. 2. ed. São Paulo: Centauro, 2006.

HALL, Stuart. Cultural Identity and Diaspora. In: RUTHERFORD, Jonathan (ed.). Identity: Community, Culture, Difference. London: Lawrence \& Wishart, 1990.

HOWELLS, Coral Ann. Not belonging, but Longing: shifts of Emphasis in Contemporary Diasporic Writing in English Canada. In: (ed.). MAVER, Igor. Diasporic subjectivity and cultural brokering in contemporary post-colonial literatures. United Kingdom: Lexington Books, 2009.

HUA, Anh. Diaspora and cultural memory. In: AGNEW, Vijay (ed.). Diaspora, memory and identity: a search for home. Toronto, Canada: University of Toronto Press, 2008.

ISHII, Satoshi. Enryo-Sasshi Communication: A Key to Understanding Japanese Interpersonal Relations. Cross Currents, 11(1): 49-58, 1984.

JOHNSTON, Genevieve Fuji; ENOMOTO, Randy (ed.). Race, Racialization, and Antiracism in Canada and beyond. Toronto, Canada: University of Toronto Press, 2007.

KOGAWA, Joy. Obasan. Harmondsworth: Penguin, 1983.

LEBRA, Takie Sugiyama. Japanese patterns of behavior. Honolulu: University of Hawaii Press, 1976.

LEE-LOY, Anne - Marie. Asian American Mothering in the Absence of Talk Story: Obasan and Chorus of Mushrooms. In: PODNIEKS, Elizabeth; O'REILLY, Andrea (eds.). Textual Mothers/Maternal Texts: motherhood in contemporary women's literatures. Ontario: Wilfrid University Press, 2010.

LITTLE, Bliss; BROOME, Benjamin. Diaspora. In: JACKSON, Ronald; HOGG, Michael (eds.). Encyclopedia of identity. Thousand Oaks: SAGE Publications, 2010.

MIIKE, Yoshitaka. Enryo. In: JACKSON II, Ronald L. (ed). Encyclopedia of Identity. vol. 1. London: Sage, 2010.

POUTIGNAT, Philippe; STREIFF-FENART, Jocelyne. Teorias da etnicidade: seguido de grupos étnicos e suas fronteiras de Fredrik Barth. Tradução Elcio Fernandes. 2. ed. São Paulo: Unesp, 2011.

SAID, Edward. Cultura e imperialismo. Tradução Denise Bottmann. São Paulo: Companhia das Letras, 2011. 
SANTOS, Boaventura Souza. Pela mão de Alice: o social e o político na pós-modernidade. 6. ed. São Paulo: Cortez, 1999.

SELBY, Sharon. Memory and identity in Canadian fiction. Jefferson: McFarland \& Company, Inc., 2018.

TAYLOR, Charles. The Politics of Recognition. In: GUTMAN, Amy (ed). Multiculturalism. New Jersey: Princeton University Press, 1994.

VERDUYN, Christl; TY, Eleanor (eds.). Asian Canadian Writing beyond auto ethnography. Ontario: Wilfrid Laurier University Press, 2008.

WELSCH, Wolfgang. Transculturality: the puzzling form of cultures today In: FEATHERSTONE, Mike; LASH, Scott (eds). Spaces of Culture: City, Nation, World. London: Sage, 1999.

WIERZBICKA, Anna. Japanese key words and core cultural values. Language in Society. Vol. 123. no. 3, 333-385, 1991.

WOODWARD, Kathryn. Identidade e diferença: uma introdução teórica e conceitual. In: SILVA, Tomaz Tadeu da. (org.). Identidade e diferença: a perspectiva dos estudos culturais. Tradução Tomaz Tadeu da Silva. 11. ed. Petrópolis: Vozes, 2012.

ZHANG, Benzi. Asian Diaspora Poetry in North America. New York: Routledge, Taylor and Francis group, 2008.

\section{Notas}

1 Professora Doutora da Universidade Federal da Paraíba, Campus IV, Mamanguape, Paraíba. Email: mdrsl@uol.com.br. Esse artigo apresenta um recorte de sua tese, orientada pela Professora Liane Schneider, aqui, coautora do artigo.

2 Professora Doutora da Universidade Federal da Paraíba, Campus I, Professora Titular do DLEM/UFPB, email: schliane@gmail.com.

3 "Asian Canadian literature is not simply a multicultural subsection of something bigger called Canadian literature. It is more than just a piece of the Canadian literary puzzle. Rather, it situates Canadian literature within a complex and delicate global network of routes and passages that are at once cultural and historical".

4 "by the recognition of a necessary heterogeneity and diversity, by a conception of 'identity' which lives with and through, not despite, difference; by hybridity. Diaspora identities are those which are constantly producing and reproducing themselves anew, through transformation and difference".

5 "the turn to diaspora signals a demand for finding a way to speak about the complexities of connections between communities, of the unredressed griefs and disarticulated longings from which collectivities emerge".

6 "to turn to diaspora is to turn to the power of relation and the enabling possibilities of difference. To turn to diaspora is to turn away from the seemingly inexorable march of history and towards the secret of memories embedded within the intimacies of the everyday".

7 "since the late 1960s, diaspora has been used to designate many different groups of people, including ethnic and racial minorities [...]. In addition to referring to groups of people, the term diaspora has also been used to refer to the dispersion of a language or culture or any object that is scattered from its origin or center".

8 " [...] unless one is satisfied with a simple statistical, a temporal, and unifying reality, 'diaspora often fails to present the workings of the thing it ought to best describe: the relationship to what I call a "referent origin"".

$9 \quad$ "Minority marks a relation defined by racialization and experienced as diaspora. That is, Asian Canadian literature, for example, relates as a minor literature to Canadian literature not because it is less important, valuable, and illuminating, but because it cannot be divorced from the long histories of racialization that mark Asian Canadian communities as being in the minority in relation to a dominant culture. In this 
experience of racialization, Asian Canadian communities are marked both by a fierce sense of belonging to this country, of having helped to build it, and by histories of dislocation that sustain a sense of belonging that exceeds the nation-state".

10 "not always as an entity of consistency or immanence, but rather a realm that multiplies connections and absorbs new meanings. [...] memory, as a result, should be considered as a becoming that struggles to recuperate new relationships between the past and the present".

11 "Episodic memory is identified as the seat of subjectivity".

12 "Kick all the Japs out".

13 Just at the last minute, before aunt Emily walked out to the plane, she asked me if I really wanted to "know everything". "I'll send you some of my correspondence and stuff. Would you like that?" she asked.

"Sure," I lied.

Now here I am this autumn morning, five months later, with her heavy package on my lap. Many of the conference papers she showed me last May are here as well as her diary and a bulging manila envelope full of letters. [...] Obasan bends over to pick up the letters and hands me them open to me one at a time. There's a copy of a letter to Mackenzie King; one to Mr. glen, Minister of Mines and Resources; one from General Headquarters Supreme Commander for the Allied Powers; [...] All the details of death that are left in the laps of the living.

14 “'”this diary of Aunt Emily's is the largest I have ever seen".

15 "the book feels heavy with the voices from the past - a connection to Mother and Grandma Kato I did not know existed".

16 "I fell like a burglar as I read, breaking into a private house only to discover it's my childhood house filled with corners and rooms I've never seen".

17 "I am sometimes not certain whether it is a cluttered attic in which I sit, a waiting room, a tunnel, a train. There is no beginning and no end to the forest, or the dust storm, no edge from which to know where the clearing begins. Here, in this familiar density, beneath this cloak, within this carapace, is the longing within the darkness".

18 O ritual de banho japonês possui quatro etapas. Sentado em um banquinho baixo, o banhista usa um balde para lavar a sujeira com água quente esvaziando-o a partir do banho preparado, em seguida, desce para a banheira para uma primeira imersão. Retornando, o banhista se senta no banquinho e se esfrega com sabão e bucha, em seguida, enxagua-se bem. O passo final é um relaxante, segundo mergulhar na banheira.

19 The Japanese bathing ritual has four steps. Seated on a low stool, the bather uses a bucket to rinse away dirt with hot water scooped from the prepared bath, then descends into the tub for a first soak. Reemerging, the bather sits on the stool and scrubs with soap and loofah, then rinses thoroughly. The final step is a relaxing, second soak in the tub. http://www.motherearthliving.com/green-living/the-water-cure. aspx\#ixzz3P2k1JLZU (acesso 11/12/2014)

19 "public baths and bathing have particular cultural resonance to the Japanese, not only as a matter of hygiene, but because bathhouses are understood to be sites in which cultural values and socialization can be practised and a more culturally specific "group think" mentality expressed".

20 "we are one flesh, one Family, washing each other or submerged in hot water".

21 Autor do texto: Enryo-Sasshi Communication: A Key to Understanding Japanese Interpersonal Relations (1984).

22 Enryo is a product of the suppression of individuality under the pressure of group solidarity and conformity, empathetic considerations for other's convenience or comfort, concern to prevent ego's own embarrassment, and the wish to maintain ego's freedom by avoiding social involvement without hurting. (LEBRA, p. 252, 1976)

23 "I squat slowly at the other end of the tub. This is sweet torture, with Gradma happy and approving and enjoying the heat I cannot endure. I am more brave, more praiseworthy than Stephen. He will not bathe with Grandma. But I will suffer endless indignities of the flesh for the pleasure of my grandmother's pleasure. [...] My body is extended beside hers and she makes waves to cover my shoulders. Once the body is fully immersed, there is a torpid peace [...] At some point, Grandma has opened her eyes and rolled her washcloth into a tight damp fist. I stand beside her and over the redness of my body she scrubs vigorously, like an eraser over the dirty page. [...] she rubs each of my fingers, my hands, arms, chest, belly and abdomen, neck, back, buttocks, tights, legs [...] she soaps me thoroughly and I am rinsed off before she soaps herself. [...] I am dressed in a nemaki, a sleeping garment of purple and white cotton which Grandma has sewn by hand. It has rectangular envelope-shaped sleeves with openings at the armpits and two ties meeting in a bow at the back. I am supremely safe in my nemaki, under the heavy bright-coloured futon in my house". 
"Indeed, the most memorable depiction of bathing is the silent bathhouse in Slocan when Naomi and Obasan are rejected by the other women in the community. Significantly, in this episode, silence actually converges with cultural practice to fragment rather than fortify an ethnic identity".

25 "when I look back, she acknowledges me with a crooked unfriendly smile but keeps nodding to the girls. For some reason she is disapproving of me".

26 "the two women are whispering to Reiko and Yuri and avoiding all contact with us, greeting us neither formally or informally. Obasan and Nomura - obasan also have their heads down. Yuri, who has been swimming around and calling to me, stops and looks at me oddly. What, I wonder, can it be?".

27 Traditional Japanese customs regarding health and health care are very different from the medical mores of Westerners. The concept of "hazukashii" or shame, is linked to all aspects of Japanese life: family, business and health. Great importance is placed on achieving success and maintaining health and close family ties. Poor health can be a very shameful experience for the Japanese. http://classroom.synonym. com/healthcare-beliefs-japanese-12859.html (acesso 11/12/2014)

28 "I stay in the corner alone, watching the two women whispering with their eyes lowered".

29 "is a word warrior".

30 "for the rest of the car ride home I kept quiet while Aunt Emily bulldozed on. I could see that we were in for an evening of marathon talk, whether anyone else felt up to it or not".

31 Grifo nosso.

32 “'she's not like them", Stephen says, jerking his thumb at Uncle and Obasan".

33 "functions as a psychological filter and requires control of one's thoughts and actions so as to avoid carelessly saying or doing things".

34 "mother's voice is yasashi, soft, tender in the dimming daylight. She is altogether yasashi".

35 "Obasan teaches me not to be wagamama by always heeding everyone's needs".

36 "Stephen has made himself altogether unfamiliar with speaking Japanese" (KOGAWA, 1983, p. 231).

37 “"what a serious baby - fed on milk and Momotaro". "Milk and Momotaro?" I asked. "Culture clash?"”.

38 People who have retained the normal intolerance of lactose include: Chinese, Japanese, Inuit, native Americans, Australian Aborigines, Iranians, Lebanese and many African tribes including the Zulus, Xhosas and Swazis. These people, generally, do not have a history of pastoralism. http://www.whitelies. org.uk/health-nutrition/lactose-intolerance (acesso em 11/12/2014)

39 "he is always uncomfortable when anything is "too Japanese"".

40 "In Miss Langston's grades seven and eight class, I have a hard time living up to his reputation. He was her favourite student. Sometimes I wonder if she is so disappointed in me that she marks me extra hard. Her red X's on my papers are like scratches and wounds. "Not like your brother, are you?" She said once, returning a poor paper".

41 "sketches a different picture of the relation between cultures. Not one of isolation and of conflict, but one of entanglement, intermixing and commonness. It promotes not separation, but exchange and interaction".

42 "Trans in relation to cultures refers also to acknowledging common values and purposes that 'transcend" the margins of particular cultures. Transculturalism involves looking at oneself in cultural disguise, through the eyes of others. This process, when happening in circunstances of mutual human respect, cannot but imply a degree of liberation from cultural fixities, from the idea of mutually in actualizing and materializing multiple identifications. [...] the cement of heterogeneous societies must involve ongoing negotiation and revisability of notions of collective and individual rights and responsabilities among dominant and dissident groups".

43 "Visually identified as "foreigners" and submitted hence to racialization, the members of "visible minorities" in these stories problematize dominant definitions of the Canadian multicultural nation that still now, in the twenty-first century, rests heavily on its colonial past". (DOMÍNGUEZ; LUCAS; LÓPEZ, 2011, p. 43)

44 "The girl with the long ringlets who sits in front of Stephen said to him, "All the japs kids at school are going to be sent away and they're bad and you're a Jap". And so, Stephen tells me, am I. "Are we father? I ask Father. "No," Father says. "We're Canadian". It is a riddle, Stephen tells me. We are both the enemy and no the enemy".

45 "all the teachers and children in our school are Japanese. The white children in Slocan go to a different school". "along the edge of the town, then into the trees and up along the thin ridges into a tunnel and we are on our way again. Clackity clack, clackity clack, clackity clack, so long, Slocan".

47 "it is obvious that, by its very organization of social communities in "race" and ethnic terms, the state constantly creates "Canadians" and "others". 
48 "Framed by the discourse of multiculturalism, under which skin of colour is used to identify individuals as either Canadian or 'Other'. In this form of racialization, individuals are assigned a racial identity by parents, by ethnic or racial identified community members, or by members of general society. They then grow up to accept or reject this identity. Such constructions of identity emerge as a response to the racism and racialization to which those identified as 'Other' Canadians are subjected".

49 Recent works by ethnic, multicultural, or minority writers in Canada have become more diverse and experimental in form, theme, focus, and technique".

50 "whiteness has been naturalized as the norm for English Canadian cultural identity".

51 "memory is a key to personal, social, and cultural history and is dependent on time and context. What we remember is articulated by the major political and social narratives of our times, they enable us to reconstruct history by identifying and filling gaps in its many narratives".

52 "as a condition of subjectivity".

53 "These memorial acts of transfer involve the dynamic negotiation between past and present, the individual and the collective, the public and private, recalling and forgetting, power and powerlessness, history and myth, trauma and nostalgia, consciousness and unconsciousness, fears and desires".

54 "Once, when Penny brought me to their door, her mother said through her small precise mouth, "Penny, I told you, I told you", and closed the door leaving me alone outside".

55 "cultural or collective memory constitutes the collective memory of many, encompassing generations". 